\title{
Tecnologias digitais na prevenção de pé diabético: uma revisão sobre aplicativos móveis
}

\section{Digital technologies in the prevention of diabetic foot: a review on mobile applications}

\section{Tecnologías digitales en la prevención del pie diabético: una revisión sobre aplicaciones móviles}

\author{
Sabado Gomes Dabó1, Maria Girlane Sousa Albuquerque Brandão ${ }^{1 *}$, Thiago Moura de Araújo', \\ Natasha Marques Frota', Vivian Saraiva Veras ${ }^{1}$
}

ORCID IDS

Dabó SG (D) https://orcid.org/0000-0002-2782-2106

Brandão MGSA (D) https://orcid.org/0000-0002-9925-4750

Araújo TM (D) http://orcid.org/0000-0002-8410-0337

Frota NM (D) https://orcid.org/0000-0001-8307-6542

Veras VS (D) https://orcid.org/0000-0003-3267-3712

\section{COMO CITAR}

Dabó SG; Brandão MGSA; Araújo TM; Frota NM; Veras VS. Tecnologias digitais na prevenção de pé diabético: uma revisão sobre aplicativos móveis. ESTIMA, Braz. J. Enterostomal Ther., 18, 2020: e1420. https:// doi.org/10.30886/estima.v18.870_PT

\section{RESUMO}

Objetivo: Analisar aplicativos móveis desenvolvidos para prevenção do pé diabético. Método: Revisão integrativa, com buscas nas bases de dados LILACS, BDENF, Scopus, Web of Science e PubMed, no período de 2000 a 2019. Após critérios de elegibilidade, a amostra constituiu-se de nove artigos. Resultados: Os aplicativos móveis para prevenção do pé diabético, se baseiam no monitoramento online dos pés por meio de imagens, avaliação de imagens térmicas dos pés, captura de imagens da planta do pé, recomendações de autocuidado com os pés e classificação do risco de pé diabético. A análise dos artigos evidencia que os aplicativos foram considerados como boa estratégia de prevenção. Conclusão: A realização deste estudo possibilitou a identificação de nove aplicativos móveis desenvolvidos para prevenção do pé diabético, com predomínio no uso da termometria como principal medida para prevenção e detecção precoce das úlceras do pé diabético, com utilização de imagens térmicas e sensores associados ao aplicativo móvel.

DESCRITORES: Aplicativos móveis. Diabetes mellitus. Pé diabético. Estomaterapia.

\begin{abstract}
Objective: Analyze mobile applications developed for prevention of diabetic foot. Method: Integrative review, with searches in LILACS, BDENF, Scopus, Web of Science and PubMed databases, from 2000 to 2019. After eligibility criteria, the sample consisted of nine articles. Results: The mobile applications for prevention of diabetic foot are based on online foot monitoring through images, evaluation of thermal images of the feet, capture of images of the sole of the foot, recommendations for self-care with the feet and classification of the risk of diabetic foot. The analysis of the articles shows that the applications were considered a good prevention strategy. Conclusion: This study enabled the identification of nine mobile applications developed for prevention of diabetic foot, with predominance in the use of thermometry as the main measure for prevention and early detection of diabetic foot ulcers, with the use of thermal images and sensors associated with the mobile application.
\end{abstract}

DESCRIPTORS: Mobile applications. Diabetes mellitus. Diabetic foot. Enterostomal therapy.

1. Universidade da Integração Internacional da Lusofonia Afro-Brasileira - Departamento de Enfermagem - Redenção (CE), Brasil. *Autora correspondente: girlane.albuquerque@yahoo.com.br

Recebido: Mar. 17, 2020 | Aceito: Abr. 30, 2020 


\section{RESUMEN}

Objetivo: Analizar aplicaciones móviles desarrolladas para la prevención del pie diabético. Método: Revisión Integrativa, con búsquedas en las bases de datos LILACS, BDENF, SCOPUS, Web of Science y PubMed, en el período 2000 a 2019. Después de los criterios de elegibilidad, la muestra consistió en nueve artículos. Resultados: Las aplicaciones móviles para la prevención del pie diabético se basan en el monitoreo en línea de los pies mediante imágenes, evaluación de imágenes térmicas de los pies, captura de imágenes del pie, recomendaciones para el autocuidado de los pies y clasificación del riesgo de pie. diabético El análisis de los artículos muestra que las aplicaciones se consideraron una buena estrategia de prevención. Conclusión: El desempeño de este estudio permitió la identificación de nueve aplicaciones móviles desarrolladas para la prevención del pie diabético, con un predominio en el uso de la termometría como la medida principal para la prevención y detección temprana de las úlceras del pie diabético, con el uso de fotografías térmicas y sensores asociados con la aplicación móvil.

DESCRIPTORES: Aplicaciones móviles. Diabetes mellitus. Pie diabético. Estomaterapia.

\section{INTRODUÇÃO}

As complicações do Diabetes mellitus (DM) podem ser macro e microvasculares e, na maioria das vezes, acontecem pela dificuldade em manter as taxas glicêmicas sob controle ${ }^{1}$. Entre as complicações, a neuropatia diabética é a mais frequente, caracterizada por síndromes clínicas que afetam diferentes partes do sistema nervoso e apresentam diversas manifestações clínicas, que têm como principal desfecho as úlceras nos pés, que, associadas às isquemias, deformidades ou infecções, induzem ao chamado pé diabético ${ }^{2}$.

O pé diabético promove diminuição da qualidade de vida e perda da mobilidade física, e, na maioria dos casos, pode culminar em amputações de membros inferiores e cerca de 40 e $60 \%$ de amputações não traumáticas ${ }^{3}$.

Por ser uma doença crônica de controle eminentemente ambulatorial, a educação em saúde possui papel fundamental na prevenção de complicações do DM. Assim, pessoas com DM precisam receber rotineiramente instruções sobre autocuidado preventivo de lesões nos pés e cuidados podológicos regulares ${ }^{2}$.

O uso das tecnologias de informação e comunicação (TICs) pode ser uma forte aliada no processo de educação em saúde para pessoas com DM na prevenção do pé diabético, frente à existência de aplicações móveis que podem auxiliar no empoderamento dos usuários, melhorar o suporte dos cuidados em saúde, aumentar a eficiência no autocuidado e a qualidade de vida ${ }^{4}$.

Destarte, diversos aplicativos móveis estão sendo desenvolvidos e validados, inclusive aplicativos para o autocuidado e o automonitoramento de pessoas com DM,com funcionalidades que ajudam na supervisão diária dos pés, na acessibilidade a tratamentos, educação em saúde, exames e diagnósticos, que podem combinar a utilização de imagens, vídeos, áudios e linguagem acessível, o que aumenta as oportunidades e a motivação para prevenção do pé diabético ${ }^{5-7}$.

Os aplicativos móveis podem oferecer aos enfermeiros a oportunidade de estreitarem laços com seus clientes com DM e familiares, e orientá-los para o autocuidado, aprimorar seu conhecimento científico, auxiliar durante a consulta de enfermagem na prevenção de riscos, monitoramento e controle de complicações, e assim, ampliar as chances de prevenção e deteç̧ão precoce de alterações nos pés 5 .

A partir da evolução das ferramentas tecnológicas e da ascensão das tecnologias em saúde, os enfermeiros e os demais profissionais de saúde devem atualizar-se para conhecer essas tecnologias para educação, prevenção e promoção da saúde de pessoas com DM e incrementar os cuidados de saúde dos usuários.

O objetivo deste estudo é analisar os aplicativos móveis desenvolvidos para prevenção do pé diabético.

\section{MÉTODO}

Estudo do tipo revisão integrativa (RI), método de pesquisa que permite a síntese e as conclusões gerais do estado do conhecimento sobre determinado tema, além de possibilitar suporte para a tomada de decisão e a melhoria da prática clínica, e, assim, apontar possíveis lacunas que implicam na realização de novos estudos na área temática em questão ${ }^{8}$.

A condução da RI seguiu seis etapas: identificação do tema e seleção da questão de pesquisa; estabelecimento de critérios para inclusão e exclusão de estudos; categorização dos estudos; avaliação dos estudos incluídos na revisão integrativa; interpretação dos resultados e síntese do conhecimento dos principais resultados evidenciados na análise dos artigos incluídos ${ }^{9}$. 
A questão de pesquisa foi elaborada de acordo com a estratégica PICO, acrônimo no idioma inglês que significa "paciente, intervenção, comparação e resultados". Ela representa um importante método para a elaboração da pergunta norteadora e posterior busca de evidências na literatura ${ }^{10}$. Com base nessa estratégia, a presente revisão considerou "P" os pacientes com diabetes, "I" se referiu à utilização de aplicativos móveis, "C" não houve palavras correspondentes, e "O” se associou ao monitoramento e prevenção do pé diabético.

Dessa forma, a seguinte pergunta norteadora foi formulada: quais aplicativos móveis disponíveis na literatura foram desenvolvidos para prevenção do pé diabético?

O levantamento bibliográfico ocorreu no período de outubro de 2019 a janeiro de 2020 nas seguintes bases de dados: Literatura Latino-Americana e do Caribe em Ciências da Saúde (LILACS), a Base de Dados de Enfermagem (BDENF), SCOPUS, Web of Science e PubMed. Foi realizado o cruzamento dos descritores Aplicativos Móveis (Mobile Applications) AND Diabetes Mellitus (Diabetes Mellitus) AND Pé Diabético (Diabetic Foot), extraídos do DeCS (Descritor em Ciências da Saúde) e do MeSH (Medical Subject Headings).

Elegeram-se como critérios de inclusão dos estudos: artigos disponíveis na íntegra, nos idiomas português, inglês e espanhol, que respondessem à questão norteadora do estudo, publicados no período entre 2009 a 2019. Os critérios de exclusão foram publicações repetidas, editoriais, manuais, livros, teses, dissertações, monografias e estudos de revisão. Foi utilizado instrumento adaptado de Ursi ${ }^{11}$ para extração dos artigos. $\mathrm{O}$ instrumento permite a obtenção de informações sobre a identificação do artigo (título, idioma, ano de publicação, nome do periódico), características metodológicas do estudo, das intervenções mensuradas e dos resultados encontrados e dos níveis de evidência.

Ao se realizar a busca dos artigos, encontraram-se 297 artigos na PubMed, 10 na Scopus, 9 na Web of Science, 3 na LILACS e 3 na BDENF, totalizando 322 artigos. Em seguida, foi realizada uma pré-seleção dos artigos localizados nas buscas efetuadas por meio da leitura do título e do resumo. Excluíram-se 3 artigos por não estarem disponíveis, 16 trabalhos de congressos, 24 revisões de literatura, 4 livros e 100 artigos por não se adequarem aos critérios de inclusão.

Efetivou-se leitura criteriosa na íntegra dos 175 artigos restantes, com objetivo de identificar ideias-chave e orientações direcionadas ao objeto de estudo. Desses, foram excluídos 13 estudos repetidos e 153 que não respondem à questão norteadora, o que proporcionou uma amostra final de 9 estudos, conforme o PRISMA (Fig. 1). As etapas de seleção dos estudos foram realizadas por dois autores da revisão de forma independente.

Realizou-se, ainda, uma terceira leitura para confirmar as informações obtidas na segunda leitura e aprofundar a análise de cada manuscrito incluído na pesquisa.

A Tabela 1 apresenta a justificativa de exclusão dos artigos após identificação nas bases e a leitura na íntegra até a amostra final de nove artigos.

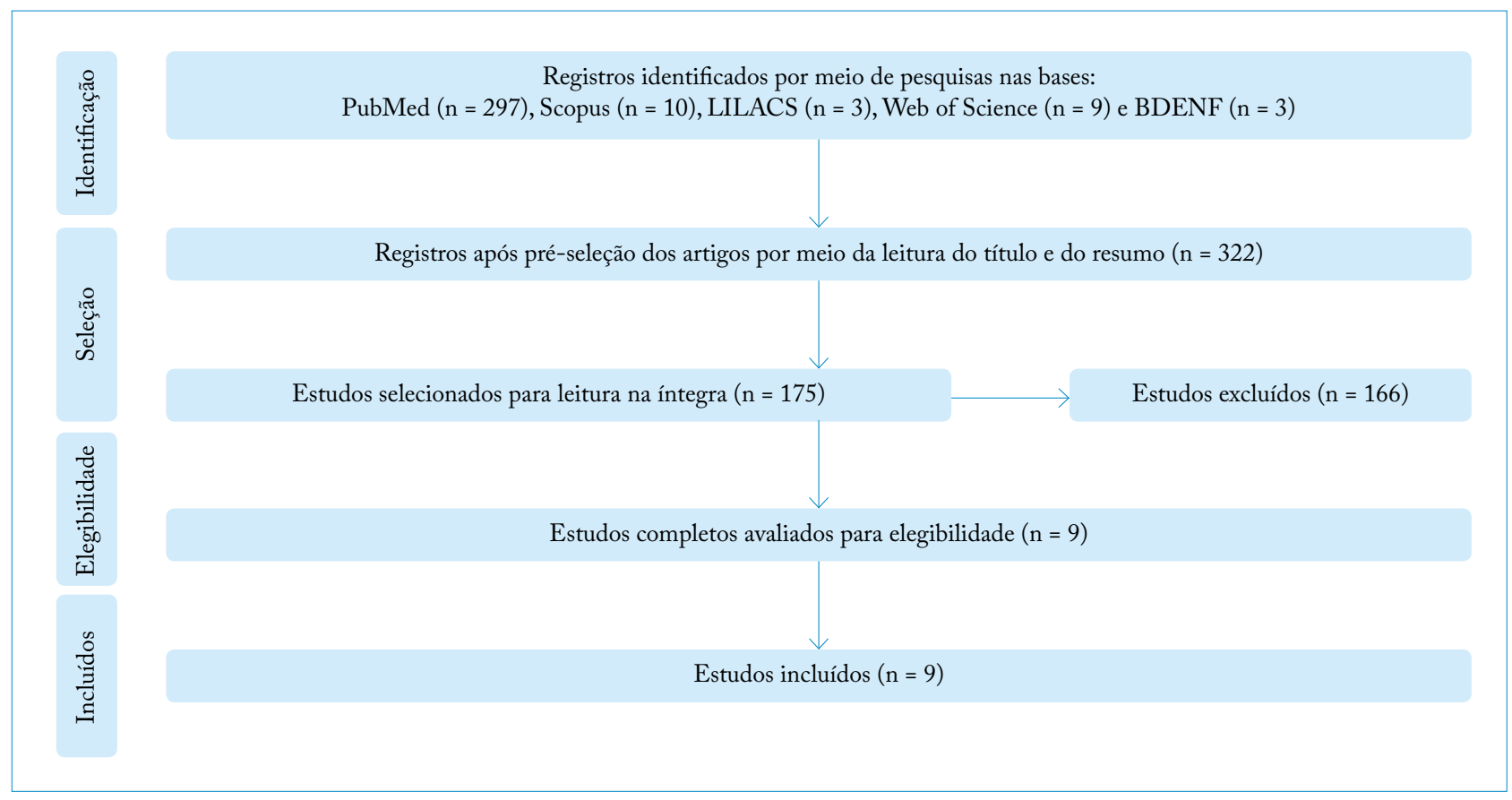

Figura 1. Fluxograma da seleção dos estudos segundo o Preferred Reporting Items for Systematic Reviews and Meta-Analyses (PRISMA 2009). 
Tabela 1. Justificativa de exclusão dos artigos após a leitura na íntegra.

\begin{tabular}{|c|c|c|c|c|c|c|}
\hline Artigos & LILACS & Scopus & $\begin{array}{l}\text { Web of } \\
\text { Science }\end{array}$ & BDENF & PubMed & Total \\
\hline Selecionados & 03 & 10 & 09 & 03 & 150 & 175 \\
\hline Não se adequa & 01 & 04 & 03 & 01 & 144 & 153 \\
\hline Repetidos & 01 & 03 & 04 & - & 05 & 13 \\
\hline Amostra Final & 01 & - & 02 & - & 06 & 09 \\
\hline
\end{tabular}

\section{RESULTADOS}

Os nove artigos incluídos nesta revisão integrativa estão apresentados na Tabela 2, composta por título, autoria, ano, objetivo, método, local de realização do estudo e nível de evidência. Ao analisar a qualidade das evidências científicas, percebe-se que a maioria dos artigos $(n=5)$ apresenta classificação nível VI de evidência ${ }^{12}$.

No que tange ao ano de publicação dos artigos, um foi publicado em 2013, um artigo em 2016, um publicado em 2017, quatro em 2018 e dois em 2019. Com relação ao idioma, oito artigos foram publicados em inglês. Houve diversificação no cenário de estudo, os quais foram desenvolvidos nos seguintes países: Brasil $(n=2)$, Índia $(n=1)$, Alemanha $(n=1)$, Peru $(\mathrm{n}=1)$, Holanda $(\mathrm{n}=1)$, Reino Unido $(\mathrm{n}=1)$, Emirados Árabes Unidos ( $\mathrm{n}=1)$ e Estados Unidos da América $(\mathrm{n}=1)$.

Houve predominância de estudos localizados na base de dados PubMed ( $\mathrm{n}=6)$. As bases de dados LILACS e Web of Science apresentaram, respectivamente, 1 e 2 publicações. Com relação à categoria das publicações, dois estudos foram publicados na área da enfermagem e sete estudos eram da área médica e biomédica.

Tabela 2. Distribuição dos artigos conforme título, autoria, ano, objetivo, método, local de realização do estudo e nível de evidência.

\begin{tabular}{|c|c|c|c|c|c|}
\hline $\mathrm{N}^{\circ}$ & Título & Autores/Ano & Objetivo & $\begin{array}{l}\text { Método/ } \\
\text { local }\end{array}$ & $\begin{array}{l}\text { Nível de } \\
\text { evidência }\end{array}$ \\
\hline 1 & $\begin{array}{c}\text { SoleSCAN - mobile phone } \\
\text { based monitoring of foot sole } \\
\text { for callus formation and the } \\
\text { shoe insole for pressure } \\
\text { "hot spots" }\end{array}$ & $\begin{array}{l}\text { Dutta, Dutta } \\
(2013)^{13}\end{array}$ & $\begin{array}{l}\text { Monitoramento do tecido cutâneo do } \\
\text { pé para formação de calo ou úlcera } \\
\text { para prevenção precoce. }\end{array}$ & $\begin{array}{l}\text { Ensaio clínico } \\
\text { Randomizado } \\
\text { Índia }\end{array}$ & Nível II \\
\hline 2 & $\begin{array}{l}\text { Implementation of foot } \\
\text { thermometry plus mHealth } \\
\text { to prevent diabetic foot } \\
\text { ulcers: study protocol for a } \\
\text { randomized controlled trial }\end{array}$ & $\begin{array}{l}\text { Lazo-Porras et } \\
\text { al. }(2016)^{14}\end{array}$ & $\begin{array}{l}\text { Comparar a incidência de úlcera do pé } \\
\text { diabético durante o período de estudo } \\
\text { entre aqueles que receberam só } \\
\text { termometria e aqueles que receberam } \\
\text { termometria mais mensagem de texto } \\
\text { e mensagens de voz. }\end{array}$ & $\begin{array}{l}\text { Ensaio clínico } \\
\text { Randomizado } \\
\text { Peru }\end{array}$ & Nível II \\
\hline 3 & $\begin{array}{c}\text { Mobile application for } \\
\text { ulcer detection }\end{array}$ & $\begin{array}{l}\text { Fraiwan et al. } \\
\qquad(2018)^{15}\end{array}$ & $\begin{array}{l}\text { Construir um sistema de imagem } \\
\text { térmica móvel que pode ser usado } \\
\text { como indicador para possíveis úlceras } \\
\text { em desenvolvimento. }\end{array}$ & $\begin{array}{c}\text { Estudo } \\
\text { descritivo } \\
\text { Emirados } \\
\text { Árabes Unidos }\end{array}$ & Nível VI \\
\hline 4 & $\begin{array}{l}\text { Promoting self-care of } \\
\text { diabetic foot ulcers through a } \\
\text { mobile phone app: } \\
\text { user-centered design } \\
\text { and evaluation }\end{array}$ & $\begin{array}{c}\text { Ploderer et al. } \\
(2018)^{17}\end{array}$ & $\begin{array}{l}\text { Avaliar a usabilidade e a utilidade } \\
\text { potencial de aplicativo de telefonia } \\
\text { móvel para envolver as pessoas } \\
\text { com úlceras do pé diabético } \\
\text { no autocuidado. }\end{array}$ & $\begin{array}{l}\text { Estudo } \\
\text { qualitativo } \\
\text { Holanda }\end{array}$ & Nível VI \\
\hline
\end{tabular}


Tabela 2. Continuação...

\begin{tabular}{|c|c|c|c|c|c|}
\hline $\mathrm{N}^{\circ}$ & Título & Autores/Ano & Objetivo & $\begin{array}{l}\text { Método/ } \\
\text { local }\end{array}$ & $\begin{array}{l}\text { Nível de } \\
\text { evidência }\end{array}$ \\
\hline 5 & $\begin{array}{c}\text { Continuous temperature- } \\
\text { monitoring socks } \\
\text { for home use in patients with } \\
\text { diabetes: } \\
\text { observational study }\end{array}$ & $\begin{array}{l}\text { Reyzelman et al. } \\
\qquad(2018)^{16}\end{array}$ & $\begin{array}{l}\text { Avaliar a precisão de sensores usados } \\
\text { em meias de desgaste diário, obter } \\
\text { feedback do usuário sobre } \\
\text { conforto das meias, incorporados } \\
\text { por sensores e examinar se as } \\
\text { temperaturas observadas } \\
\text { relacionam com observações clínicas. }\end{array}$ & $\begin{array}{l}\text { Estudo de caso } \\
\text { Estados Unidos }\end{array}$ & Nível VI \\
\hline 6 & $\begin{array}{c}\text { A new mobile application } \\
\text { for standardizing diabetic } \\
\text { foot images }\end{array}$ & Yap et al. $(2018)^{7}$ & $\begin{array}{l}\text { Testar a confiabilidade de aplicativo } \\
\text { quando usado com os mesmos } \\
\text { pés diabéticos em ocasiões } \\
\text { separadas e nas mãos de dois } \\
\text { operadores separados. }\end{array}$ & $\begin{array}{l}\text { Estudo } \\
\text { longitudinal } \\
\text { Reino Unido }\end{array}$ & Nível VI \\
\hline 7 & $\begin{array}{l}\text { Rehabilitation technology for } \\
\text { self-care: Customised foot and } \\
\text { ankle exercise software } \\
\text { for people } \\
\text { with diabetes }\end{array}$ & $\begin{array}{l}\text { Ferreira et al. } \\
\qquad(2019)^{19}\end{array}$ & $\begin{array}{c}\text { Desenvolver e validar o } \\
\text { conteúdo de software } \\
\text { (desktop e aplicações móveis) } \\
\text { para a autogestão e os } \\
\text { exercícios de pé e tornozelo } \\
\text { personalizados para } \\
\text { pessoas com diabetes e } \\
\text { neuropatia diabética. }\end{array}$ & $\begin{array}{c}\text { Estudo } \\
\text { quantitativo } \\
\text { Brasil }\end{array}$ & Nível VI \\
\hline
\end{tabular}

Study protocol

for a randomized controlled trial to test for preventive effects

8 of diabetic foot ulceration by telemedicine that includes sensor-equipped insoles combined with photo documentation
Investigar a hipótese de que uma gravação de duas vezes por dia de Ming et al. $(2019)^{18}$ temperaturas do pé com o auxílio da palmilha equipado com sensor (Medixfeet Insole, Thorsis Technologies $\mathrm{GmbH}$ ) pode reduzir o risco de formação de úlcera.
Ensaio clínico randomizado

Nível II Alemanha
Descrever o processo

de desenvolvimento e Mobile application for 9 evaluation of feet in people with diabetes mellitus
Vêscovi et al. $(2017)^{5}$ validação de aplicativo para

dispositivos móveis sobre avaliação e

classificação de risco dos pés de pessoas com DM.
A Tabela 3 apresenta o nome e a função dos aplicativos evidenciados na revisão e seus principais resultados. Os aplicativos móveis para prevenção do pé diabético, se baseiam no monitoramento online dos pés por meio de imagens ${ }^{13}$, avaliação de imagens térmicas dos pés ${ }^{14-16}$, captura de imagens da planta do pé ${ }^{7}$, recomendações de autocuidado com os pés ${ }^{17-19}$ e classificação do risco de pé diabético ${ }^{5}$.
A análise dos artigos evidenciam que os aplicativos foram considerados boa estratégia de prevenção, por permitir a detecção precoce de possíveis formações de calos e úlceras nos pés ${ }^{13}$, análise da temperatura dos pés de forma inovadora e barata, que possibilita intervenções precoces para prevenir o pé diabético ${ }^{14-16}$, prover a aquisição de fotos da planta do pé para observar parâmetros relevantes dos pés ${ }^{7}$, recomendações de autocuidado ${ }^{19}$, além da estratificação do risco de desenvolvimento de pé diabético ${ }^{5}$. 
Tabela 3. Distribuição dos artigos conforme autoria, nome, função do aplicativo e principais resultados.

\begin{tabular}{|c|c|c|c|}
\hline Autores & $\begin{array}{l}\text { Nome do } \\
\text { Aplicativo }\end{array}$ & Função do Aplicativo & Principais Resultados \\
\hline $\begin{array}{l}\text { Dutta, Dutta } \\
\qquad(2013)^{13}\end{array}$ & SoleSCAN & $\begin{array}{l}\text { Monitoramento online do pé por meio de } \\
\text { palmilha que registra fotos e transfere as } \\
\text { informações para um servidor de dados. }\end{array}$ & $\begin{array}{l}\text { O aplicativo foi considerado uma boa } \\
\text { estratégia de prevenção, pois permite a } \\
\text { detecção precoce de possíveis formações } \\
\text { de calos e úlceras nos pés. }\end{array}$ \\
\hline $\begin{array}{l}\text { Lazo-Porras et } \\
\text { al. }(2016)^{14}\end{array}$ & TempStat & Capturar imagens térmicas dos pés. & $\begin{array}{c}\text { A termometria pode reduzir a incidência } \\
\text { de pé diabético. O aplicativo apresentou } \\
\text { abordagem preventiva inovadora } \\
\text { e de baixo custo. }\end{array}$ \\
\hline $\begin{array}{l}\text { Fraiwan et al. } \\
\qquad(2018)^{15}\end{array}$ & FLIR ONE & $\begin{array}{l}\text { Obter imagens térmicas, processá-las e } \\
\text { analisá-las para prever ulceração. }\end{array}$ & $\begin{array}{c}\text { O sistema móvel foi bem-sucedido na } \\
\text { identificação do aumento de temperatura } \\
\text { dos pés e possibilita intervenções precoces } \\
\text { para prevenir o pé diabético. }\end{array}$ \\
\hline $\begin{array}{l}\text { Ploderer et al. } \\
\qquad(2018)^{17}\end{array}$ & My Foot Care & $\begin{array}{l}\text { Destaca metas pessoais para ajudar a } \\
\text { motivar os pacientes no autocuidado e } \\
\text { fornece lembretes para decretar o cuidado } \\
\text { regular. }\end{array}$ & $\begin{array}{l}\text { O aplicativo tem potencial generalizado na } \\
\text { prevenção e no tratamento de úlceras nos } \\
\text { pés de pessoas com diabetes. }\end{array}$ \\
\hline $\begin{array}{l}\text { Reyzelman et } \\
\text { al. }(2018)^{16}\end{array}$ & Stand-Alone & $\begin{array}{l}\text { Gerar alertas quando os pés do usuário } \\
\text { mostram aumento de temperatura. }\end{array}$ & $\begin{array}{l}\text { O monitoramento contínuo da temperatura } \\
\text { dos pés apresentou-se como uma } \\
\text { abordagem promissora de sistema de } \\
\text { alerta para intervir de maneira precoce no } \\
\text { risco de desenvolvimento de pé diabético. }\end{array}$ \\
\hline $\begin{array}{l}\text { Yap et al. } \\
(2018)^{7}\end{array}$ & FootSnap & Obter imagens da planta do pé. & $\begin{array}{l}\text { O aplicativo foi bem-sucedido em } \\
\text { padronizar a aquisição de fotos da planta } \\
\text { do pé para observar parâmetros dos pés e } \\
\text { mostrou-se significativo na prevenção e na } \\
\text { gestão de patologias do pé diabético. }\end{array}$ \\
\hline $\begin{array}{l}\text { Ferreira et al. } \\
\qquad(2019)^{19}\end{array}$ & SoPeD & $\begin{array}{l}\text { Ofertar recomendações de autocuidados } \\
\text { com os pés, diabetes, pé diabético e } \\
\text { exercícios de pé e tornozelo. }\end{array}$ & $\begin{array}{l}\text { O aplicativo foi validado e apresentou } \\
\text { alto grau de concordância entre os } \\
\text { especialistas, profissionais e usuários, } \\
\text { como aplicativo que pode ajudar na } \\
\text { prevenção do pé diabético. }\end{array}$ \\
\hline $\begin{array}{l}\text { Ming et al. } \\
(2019)^{18}\end{array}$ & SPDFA & $\begin{array}{l}\text { Medir temperaturas do pé, fornecer } \\
\text { documentação fotográfica, e avaliar o bem- } \\
\text { estar (qualidade de vida), utilizando um } \\
\text { diário interativo. }\end{array}$ & $\begin{array}{l}\text { O aplicativo provou ser uma das soluções } \\
\text { mais rentáveis para a detecção precoce de } \\
\text { úlceras do pé diabético. }\end{array}$ \\
\hline $\begin{array}{l}\text { Vêscovi et al. } \\
\qquad(2017)^{5}\end{array}$ & CuidarTech & $\begin{array}{l}\text { Avaliação e classificação do risco de pé } \\
\text { diabético. }\end{array}$ & $\begin{array}{l}\text { O aplicativo, por meio da avaliação dos } \\
\text { enfermeiros, mostrou-se confiável, } \\
\text { adequado e eficiente para classificação do } \\
\text { risco e prevenção do pé diabético. }\end{array}$ \\
\hline
\end{tabular}

\section{DISCUSSÃO}

Nesta revisão, em resposta a questão norteadora do estudo, observou-se que diferentes aplicativos desenvolvidos para prevenção de pé diabético foram apontados como satisfatórios pelos estudiosos nas publicações analisadas. Os estudos se preocuparam em investigar e inserir nos aplicativos as principais causas que levam à ulceração e elevação do risco de desenvolvimento do pé diabético.

Os aplicativos móveis apresentaram como função predominante a captura de imagens térmicas. Alguns 
aplicativos, além de realizarem termometria, enviavam alertas mediante detecção de hipertermia ${ }^{14-16}$.

O uso de aplicativos móveis com termometria pode coadjuvar a redução da incidência de úlceras que levam ao pé diabético. A termometria mede o calor superficial da pele dos pés e é uma modalidade emergente promissora e de baixo custo para avaliar e gerenciar sinais precoces de desenvolvimento de pé diabético ${ }^{14,20}$.

A maioria das complicações do pé diabético podem ser agravadas por temperaturas elevadas na planta dos pés e representar um sinal precoce de síndrome do pé diabético. Estudos realizados na Ásia e na Europa demonstraram que aplicativos móveis que realizam termometria dos pés podem ser eficientes na prevenção do pé diabético ${ }^{13,18}$ Assim, o monitoramento das temperaturas da planta do pé pode ser considerado um método eficaz para detecção precoce e possível prevenção do pé diabético ${ }^{18}$. Isso retrata a importância de aplicativos móveis que possam coadjuvar os profissionais de saúde no acompanhamento e na prevenção do surgimento de alterações que comprometam a integridade dos pés. A obtenção de imagens térmicas está aliada com a detecção de alterações plantares, que, associadas a pontos de pressão e calçados inadequados, aumentam o risco de desenvolvimento de ulcerações.

Vale destacar que os aplicativos móveis apresentam a capacidade de reunir mais de uma função. Estudo na Alemanha desenvolveu aplicativo móvel que possui além da termometria por meio de palmilha e sensores, uma estrutura que fornece toda a documentação fotográfica dos pés e avaliação do bem-estar (qualidade de vida) do usuário, por meio da utilização de diário interativo ${ }^{18}$. Percebe-se, portanto, que os aplicativos móveis podem agrupar mais de uma função e contribuir substancialmente com o monitoramento dos pés, no intuito de prevenir o surgimento do pé diabético.

Outro aplicativo desenvolvido na Índia apresentou como função primordial o monitoramento online dos pés por meio de palmilhas que captam imagens e as enviam para um servidor de dados, que pode ser acessado por profissionais de saúde ${ }^{13}$.

O monitoramento online do tecido cutâneo da planta do pé com uso de palmilha e algoritmo que processa imagens em smartphones e as enviam para profissionais de saúde pode ser um meio barato e eficaz para detectar o risco de desenvolvimento de calos e úlceras por pressão e prevenir o pé diabético ${ }^{13}$. De tal modo, o uso de palmilhas nos sapatos associados a aplicativos móveis que processam imagens pode identificar pontos de pressão e hipertermia nos pés, possibilitando, assim, intervenções precoces.

Aplicativo desenvolvido no Reino Unido para padronizar a aquisição das fotografias dos pés de pessoas com diabetes e monitoramento contínuo por profissionais de saúde foi bem-sucedido em padronizar a aquisição de fotos da planta do pé, para observar parâmetros relevantes da região plantar e mostrou-se significativo na prevenção, na gestão e no autocuidado do pé diabético ${ }^{7}$.

Dentro dessa perspectiva de autocuidado, pesquisadores da Holanda desenvolveram aplicativo móvel projetado para os usuários com DM, para motivá-los e engajá-los no autocuidado. $\mathrm{O}$ aplicativo encoraja o uso do telefone celular para tirar fotos dos pés, o qual aplica análises visuais para essas fotos e extrai informações, permitindo que os usuários e seus cuidadores acompanhem-nas. Além disso, destaca metas pessoais para ajudar a motivar os usuários com DM e fornece lembretes que encorajam o cuidado regular dos pés ${ }^{17}$.

Estudo brasileiro com escopo similar validou aplicativo móvel que permite o autocuidado e o atendimento personalizado para os pacientes por meio da oferta de recomendações de autocuidado com os pés, diabetes, pé diabético e exercícios de pé e tornozelo. Os usuários recebem recomendações online de cuidados com os pés, informações sobre DM e neuropatia diabética, autoavaliação do pé de acordo com as principais alterações (calos, deformidades e lesões), além de exercícios para os pés e tornozelos, no intuito de fortalecer os músculos e melhorar o movimento ${ }^{19}$.

Para isso, o uso de aplicativos para autogestão do DM foi positivamente associado ao comportamento de autocuidado, promovendo mudanças benéficas no estilo de vida, com capacidade de prevenção de complicações, como o pé diabético ${ }^{21}$.

A adoção de práticas educativas como estratégia no tratamento de pessoas com DM e pé em risco neuropático tem por objetivo uma perspectiva emancipatória, ou seja, de autocuidado. A referida perspectiva esteve presente na maioria dos estudos por superar o modelo curativo e ampliar o conhecimento do indivíduo sobre a doença, os hábitos de vida mais saudáveis e os cuidados regulares com os pés; isso porque, além de melhorar a qualidade de vida, aumentam sua autonomia perante a doença.

Estudo consultado infere que é necessário expandir o conhecimento e a compreensão do enfermeiro quanto à importância da avaliação dos pés das pessoas com DM e acompanhá-las em todos os níveis de atenção à saúde, além 
de instruir as pessoas com DM para o autocuidado, uma vez que a enfermagem vem passando por diversas transformações com avanços tecnológicos e deve-se aproveitar das tecnologias móveis para incrementar as práticas de promoção da saúde ${ }^{5}$.

Nessa compreensão, para coadjuvar o trabalho do enfermeiro frente ao cuidado das pessoas com DM, estudo brasileiro desenvolveu um software que fornece ao enfermeiro uma ferramenta que o auxilia na avaliação e na classificação do risco da pessoa com $\mathrm{DM}$ desenvolver pé diabético. $\mathrm{O}$ aplicativo, segundo avaliação dos juízes e enfermeiros, é funcional, confiável, adequado e eficiente para classificar o risco de desenvolver pé diabético e, assim, possibilitar a realização precoce de intervenções ${ }^{5}$. Nos cenários de saúde e tecnologia atuais existem diversas TICs que podem ajudar a determinar o risco de úlceras que culminam no pé diabético. A maioria são tecnologias portáteis, de fácil manuseio, interativas e não invasivas, que não requerem especialista para seu uso, além de permitir o acompanhamento dos cuidados com os pés dos usuários em qualquer localização geográfica. Essas ferramentas já testadas possibilitam aprimoramento com o avanço de recursos tecnológicos e aproximam o sujeito para o autocuidado, com o registro de fotos, uso de palmilhas para termometria e nas respostas aos questionários online.

Assim, diversos aplicativos em dispositivos portáteis podem facilitar a prevenção de pé diabético e melhorar a qualidade de vida de pessoas com $\mathrm{DM}^{22}$.

Novos estudos atualizados certamente são necessários para nortear tanto as decisões sobre a adoção (pelos usuários) ou recomendação (pela equipe de saúde responsável) dos aplicativos mais adequados para uso no autocuidado e na prevenção do pé diabético, como também apontar caminhos para o desenvolvimento de novas ferramentas, ou mesmo o aprimoramento das existentes, testar a usabilidade, a aceitação e o custo dos aplicativos.

Apesar de o artigo apresentar limitações, como a exclusão de artigos indisponíveis nas bases, houve apresentação de nove aplicativos móveis que podem ser utilizados por usuários e profissionais de saúde na prevenção do pé diabético.

\section{CONCLUSÃO}

A realização deste estudo possibilitou a análise de nove aplicativos móveis desenvolvidos para prevenção do pé diabético. Houve predomínio no uso da termometria como principal medida para prevenção e detecção precoce das úlceras do pé diabético, com utilização de imagens térmicas e sensores associados ao aplicativo móvel. Além da obtenção das fotos, outros aplicativos incluíram metas, instruções sobre DM e os cuidados com o pé, diário interativo, classificação de risco, exercícios de pé e tornozelo, alarmes, transferência e armazenamentos de dados.

\section{CONTRIBUIÇÃO DOS AUTORES}

Conceitualização, Dabó SG, Brandão MGSA e Veras VS; Metodologia, Dabó SG e Brandão MGSA; Investigação, Dabó SG e Brandão MGSA; Redação - Primeira versão, Dabó SG, Brandão MGSA e Veras VS; Redação - Revisão \& Edição, Dabó SG, Brandão MGSA, Araújo TM, Frota NM e Veras VS; Supervisão, Veras VS.

\section{REFERÊNCIAS}

1. Gomes DM, Dazio EMR, Paraizo CMS, Brito MVN, Gonçalves JS, Fava SMCL. Ressignificação do cuidado de uma pessoa com diabetes e pé diabético: relato de experiência. Rev Enferm Cent-Oeste Min. 2018;8:e1509. https://doi. org/10.19175/recom.v8i0.1509

2. Sociedade Brasileira de Diabetes. Diretrizes da Sociedade Brasileira de Diabetes 2019-2020. São Paulo: Clannad; 2019. [citado em 10 Jan 2020]. Disponível em: https:// www.diabetes.org.br/profissionais/images/DIRETRIZESCOMPLETA-2019-2020.pdf

3. Padilha AP, Rosa LM, Schoeller SD, Junkes C, Mendez $C B$, Martins MMFPS. Care manual for diabetic people with diabetic foot: construction by Scoping Study. Texto Contexto - Enferm. 2018;26(4):e2190017. https://doi. org/10.1590/0104-07072017002190017

4. Chaves ASC, Oliveira GM, Jesus LMS, Martins JL, Silva VC. Uso de aplicativos para dispositivos móveis no processo de educação em saúde: reflexos da contemporaneidade. Rev Humanid Inov. 2018;5(6):34-42. https://doi.org/10.20873/ uft.2447-4266.2018v4n6p826

5. Vêscovi SJB, Caniçali Primo C, Sant'Anna HC, Bringuete MEO, Rohr RV, Prado TN, et al. Mobile application for evaluation of feet in people with diabetes mellitus. Acta Paul Enferm. 2017;30(6):60713. https://doi.org/10.1590/1982-0194201700087 
6. Scarcella MFS. Elaboração e desenvolvimento de aplicativo móvel para autocuidado e automonitoramento do pé diabético [Dissertação]. [Belo Horizonte]: Universidade Federal de Minas Gerais; 2017.

7. Yap $\mathrm{MH}$, Chatwin KE, Ng C-C, Abbott CA, Bowling FL, Rajbhandaris, etal. Anewmobileapplicationfor standardizing diabetic foot images. J Diabetes Sci Technol. 2018;12(1):16973. https://doi.org/10.1177/1932296817713761

8. Correia BC, Trindade JK, Almeida AB. Fatores correlacionados à automedicação entre os jovens e adultos: uma revisão integrativa da literatura. Rev Inic Cient Ext. 2019;2(1):57-61.

9. Mendes KDS, Silveira RCCP, Galvão CM. Revisão integrativa: método de pesquisa para a incorporação de evidências na saúde e na enfermagem. Texto Contexto - Enferm. 2008;17(4):758-64. https://doi.org/10.1590/S0104-07072008000400018

10. Santos CAC, Pimenta CAM, Nobre MRC. The PICO strategy for the research question construction and evidence search. Rev Latino-Am Enfermagem. 2007;15(3):508-11. https://doi org/10.1590/s0104-11692007000300023

11. Ursi ES. Prevenção de lesões de pele no perioperatório: revisão integrativa da literatura [Dissertação]. [Ribeirão Preto]: Universidade de São Paulo; 2005.

12. Polit DF, Beck CT. Fundamentos de Pesquisa em Enfermagem: Avaliação de Evidências para a Prática da Enfermagem. $7^{\text {a }}$ ed. Porto Alegre: Artmed; 2011.

13. Dutta A, Dutta A. SoleSCAN - mobile phone based monitoring of foot sole for callus formation and the shoe insole for pressure "hot spots". 2013 IEEE Point-of-Care Healthcare Technologies (PHT); 2013 Jan. 16-18; Bangalore: IEEE; 2013. https://doi.org/10.1109/PHT.2013.6461354

14. Lazo-Porras M, Bernabe-Ortiz A, Sacksteder KA, Gilman $\mathrm{RH}$, Malaga $\mathrm{G}$, Armstrong DG, et al. Implementation of foot thermometry plus mHealth to prevent diabetic foot ulcers: study protocol for a randomized controlled trial. Trials. 2016;17:206. https://doi.org/10.1186/s13063-016-1333-1
15. Fraiwan L, Ninan J, Al-Khodari M. Mobile application for ulcer detection. Open Biomed Eng J. 2018;12:16-26. https://doi. org/10.2174/1874120701812010016

16. Reyzelman AM, Koelewyn K, Murphy M, Shen X, Yu E, Pillai $\mathrm{R}$, et al. Continuous temperature-monitoring socks for home use in patients with diabetes: observational study. J Med Internet Res. 2018;20(12):e12460. https://doi. $\operatorname{org} / 10.2196 / 12460$

17. Ploderer B, Brown R, Seng LSD, Lazzarini PA, van Netten Jj. Promoting self-care of diabetic foot ulcers through a mobile phone app: user-centered design and evaluation. JMIR Diabetes. 2018;3(4):e10105. https://doi.org/10.2196/10105

18. Ming A, Walter I, Alhajjar A, Leuckert M, Mertens PR. Study protocol for a randomized controlled trial to test for preventive effects of diabetic foot ulceration by telemedicine that includes sensor-equipped insoles combined with photo documentation. Trials. 2019;20:521. https://doi. org/10.1186/s13063-019-3623-x

19. Ferreira JSSP, Sacco ICN, Siqueira AA, Almeida MHM, Sartor CD. Rehabilitation technology for self-care: Customised foot and ankle exercise software for people with diabetes. PLoS ONE. 2019;14(6):e0218560. https://doi.org/10.1371/journal. pone. 0218560

20. Skafjeld A, Iversen MM, Holme I, Ribu L, Hvaal K, Kilhovd BK. A pilot study testing the feasibility of skin temperature monitoring to reduce recurrent foot ulcers in patients with diabetes - a randomized controlled trial. BMC Endocr Disord. 2015;15:55. https://doi.org/10.1186/s12902-015-0054-x

21. Kebede MM, Pischke CR. Popular diabetes apps and the impact of diabetes app use on self-care behaviour: A survey among the digital community of persons with diabetes on social media. Front Endocrinol. 2019;10:135. https://doi. org/10.3389/fendo.2019.00135

22. Martín-Vaquero J, Encinas AH, Queiruga-Dios A, Bullón J, Martínez-Nova A, González JT, et al. Review on wearables to monitor foot temperature in diabetic patients. Sensors. 2019;19(4):776. https://doi.org/10.3390/s19040776 